Math. Model. Nat. Phenom.

Vol. 8, No. 5, 2013, pp. 190-205

DOI: $10.1051 / \mathrm{mmnp} / 20138512$

\title{
On Abrikosov Lattice Solutions of the Ginzburg-Landau Equations
}

\author{
T. Tzaneteas ${ }^{1 *}$, I. M. Sigal ${ }^{2 * *}$ \\ ${ }^{1}$ Department of Mathematics, Aarhus University, Aarhus, Denmark \\ ${ }^{2}$ Dept. of Mathematics, Univ. of Toronto, Toronto, Canada, M5S 2E4
}

\begin{abstract}
Building on earlier work, we have given in [29] a proof of existence of Abrikosov vortex lattices in the Ginzburg-Landau model of superconductivity and shown that the triangular lattice gives the lowest energy per lattice cell. After [29] was published, we realized that it proves a stronger result than was stated there. This result is recorded in the present paper. The proofs remain the same as in [29], apart from some streamlining.
\end{abstract}

Keywords and phrases: magnetic vortices, superconductivity, Ginzburg-Landau equations, Abrikosov vortex lattices, bifurcation

Mathematics Subject Classification: 35Q56, 35B32

\section{Introduction}

1.1 The Ginzburg-Landau equations. The Ginzburg-Landau model of superconductivity describes a superconductor contained in $\Omega \subset \mathbb{R}^{n}, n=2$ or 3 , in terms of a complex order parameter $\Psi: \Omega \rightarrow \mathbb{C}$, and a magnetic potential $A: \Omega \rightarrow \mathbb{R}^{n 1}$. The key physical quantities for the model are

- the density of superconducting pairs of electrons, $n_{s}:=|\Psi|^{2}$;

- the magnetic field, $B:=\operatorname{curl} A$;

- and the current density, $J:=\operatorname{Im}\left(\bar{\Psi} \nabla_{A} \Psi\right)$.

In the case $n=2, \operatorname{curl} A:=\frac{\partial A_{2}}{\partial x_{1}}-\frac{\partial A_{1}}{\partial x_{2}}$ is a scalar-valued function. The covariant derivative $\nabla_{A}$ is defined to be $\nabla-i A$. The Ginzburg-Landau theory specifies that the difference between the supperconducting and normal free energies in a state $(\Psi, A)$ is

$$
E_{\Omega}(\Psi, A):=\int_{\Omega}\left|\nabla_{A} \Psi\right|^{2}+|\operatorname{curl} A|^{2}+\frac{\kappa^{2}}{2}\left(1-|\Psi|^{2}\right)^{2},
$$

where $\kappa$ is a positive constant that depends on the material properties of the superconductor. (In the problem we consider here it is appropriate to deal with Helmholtz free energy at a fixed average magnetic field $b:=\frac{1}{|\Omega|} \int_{\Omega} \operatorname{curl} A$, where $|\Omega|$ is the area or volume of $\Omega$.) It follows from the Sobolev inequalities

*Corresponding author. E-mail: ttzaneteas@imf.au.dk

** Corresponding author. E-mail: im.sigal@utoronto.ca

${ }^{1}$ The Ginzburg-Landau theory is reviewed in every book on superconductivity. For reviews of rigorous results see the papers $[11,12,19]$ and the books $[16,20,26,27]$

(C) EDP Sciences, 2013 
that for bounded open sets $\Omega, \mathcal{E}_{\Omega}$ is well-defined and $C^{\infty}$ as a functional on the Sobolev space $H^{1}$. The critical points of this functional must satisfy the well-known Ginzburg-Landau equations inside $\Omega$ :

$$
\begin{gathered}
\Delta_{A} \Psi=\kappa^{2}\left(|\Psi|^{2}-1\right) \Psi, \\
\operatorname{curl}^{*} \operatorname{curl} A=\operatorname{Im}\left(\bar{\Psi} \nabla_{A} \Psi\right) .
\end{gathered}
$$

Here $\Delta_{A}=-\nabla_{A}^{*} \nabla_{A}, \nabla_{A}^{*}$ and curl* are the adjoints of $\nabla_{A}$ and curl. Explicitly, $\nabla_{A}^{*} F=-\operatorname{div} F+i A \cdot F$, and $\operatorname{curl}^{*} F=\operatorname{curl} F$ for $n=3$ and $\operatorname{curl}^{*} f=\left(\frac{\partial f}{\partial x_{2}},-\frac{\partial f}{\partial x_{1}}\right)$ for $n=2$.

It is well-known that there exists a critical value $\kappa_{c}$ (in the units used here, $\kappa_{c}=1 / \sqrt{2}$ ), that separates superconductors into two classes with different properties: Type I superconductors, which have $\kappa<\kappa_{c}$ and exhibit first-order phase transitions from the non-superconducting state to the superconducting state, and Type II superconductors, which have $\kappa>\kappa_{c}$ and exhibit second-order phase transitions and the formation of vortex lattices. Existence of these vortex lattice solutions is the subject of the present paper.

1.2 Abrikosov lattices. In 1957, Abrikosov [1] discovered solutions of (1.2) in $n=2$ whose physical characteristics $n_{s}, B$, and $J$ are periodic with respect to a two-dimensional lattice, while independent of the third dimension, and which have a single flux per lattice cell ${ }^{2}$. (In what follows we call such solutions, with $n_{s}$ and $B$ non-constant, $\mathcal{L}$-lattice solutions, or, if a lattice $\mathcal{L}$ is fixed, lattice solutions. In physics literature they are variously called mixed states, Abrikosov mixed states, Abrikosov vortex states.) Due to an error of calculation Abrikosov concluded that the lattice which gives the minimum average energy per lattice cell ${ }^{3}$ is the square lattice. Abrikosov's error was corrected by Kleiner, Roth, and Autler [21], who showed that it is in fact the triangular lattice which minimizes the energy.

1.3 Results. In this paper we combine and extend the previous technique to give a complete and self-contained proof of the existence of Abrikosov lattice solutions. As in previous works, we consider only bulk superconductors filling all $\mathbb{R}^{3}$, with no variation along one direction, so that the problem is reduced to one on $\mathbb{R}^{2}$. To formulate our results, for a lattice $\mathcal{L} \subset \mathbb{R}^{2}$, we denote by $\Omega^{\mathcal{L}}$ and $\left|\Omega^{\mathcal{L}}\right|$ the basic lattice cell and its area, respectively (for details see Section 3). The flux quantization (see below) implies that

$$
\left|\Omega^{\mathcal{L}}\right|=\frac{2 \pi n}{b}
$$

where $b$ is the average magnetic flux per lattice cell, $b:=\frac{1}{\Omega^{\mathcal{L}}} \int_{\Omega^{\mathcal{L}}} \operatorname{curl} A$. We note that due to the reflection symmetry of the problem we can assume that $b \geq 0$. We define

$$
\kappa_{c}(\tau):=\sqrt{\frac{1}{2}\left(1-\frac{1}{\beta(\tau)}\right)},
$$

where $\beta(\tau)$ is the Abrikosov parameter, introduced in (9.1) below. We will prove the following results.

Theorem 1.1. Let $\left|b-\kappa^{2}\right| \ll 1$ and $\left(\kappa-\kappa_{c}(\tau)\right)\left(\kappa^{2}-b\right) \geq 0$. Then for every lattice $\mathcal{L}$ satisfying $(1.3)$ with $n=1$, the following holds

(I) The equations (1.2) have an $\mathcal{L}$-lattice solution in a neighbourhood of the branch of normal solutions.

(II) The above solution is unique, up to symmetry, in a neighbourhood of the normal branch.

(III) For $\left(\kappa-\kappa_{c}(\tau)\right)\left(\kappa^{2}-b\right) \neq 0$, the solution above is real analytic in $b$ in a neighbourhood of $\kappa^{2}$.

(IV) For $\kappa^{2}>1 / 2$, the lattice shape for which the average energy per lattice cell is minimized approaches the triangular lattice as $b \rightarrow \kappa^{2}$, in the sense that the shape parameter, $\tau_{\mathcal{L}}$, of $\mathcal{L}$ (see Subsection 3.3 below) approaches $\tau_{\text {triangular }}=e^{i \pi / 3}$ in $\mathbb{C}$.

\footnotetext{
${ }^{2}$ Such solutions correspond to cylindrical samples. In 2003, Abrikosov received the Nobel Prize for this discovery

${ }^{3}$ Since for lattice solutions the energy over $\mathbb{R}^{2}$ (the total energy) is infinite, one considers the average energy per lattice cell, i.e. energy per lattice cell divided by the area of the cell.
} 
Since their discovery, Abrikosov lattice solutions have been studied in numerous experimental and theoretical works. Of more mathematical studies, we mention the articles of Eilenberger [15], Lasher [22], Chapman [10] and Ovchinnikov [25].

The rigorous investigation of Abrikosov solutions began soon after their discovery. Odeh [24] sketched a proof of existence for various lattices using variational and bifurcation techniques. Barany, Golubitsky, and Turski [8] applied equivariant bifurcation theory and filled in a number of details, and Takác [28] has adapted these results to study the zeros of the bifurcating solutions. Further details and new results, in both, variational and bifurcation, approaches, were provided by [13,14]. In particular, [14] proved partial results on the relation between the bifurcation parameter and the average magnetic field $b$ (left open by previous works) and on the relation between the Ginzburg-Landau energy and the Abrikosov function, and [13] (see also [14]) found boundaries between superconducting, normal and mixed phases.

Among related results, a relation of the Ginzburg-Landau minimization problem, for a fixed, finite domain and external magnetic field, in the regime of $\kappa \rightarrow \infty$, to the Abrikosov lattice variational problem was obtained in $[3,6]$.

All the rigorous results above deal with Abrikosov lattices with one quantum of magnetic flux per lattice cell. Partial results for higher magnetic fluxes were proven in $[5,10]$.

After introducing general properties of (1.2) in Sections 2-4, we prove the above theorem in Sections $5-9$.

\section{Properties of the Ginzburg-Landau equations}

2.1 Symmetries. The Ginzburg-Landau equations exhibit a number of symmetries, that is, transformations which map solutions to solutions:

The gauge symmetry,

$$
(\Psi(x), A(x)) \mapsto\left(e^{i \eta(x)} \Psi(x), A(x)+\nabla \eta(x)\right), \quad \forall \eta \in C^{2}\left(\mathbb{R}^{2}, \mathbb{R}\right) ;
$$

The translation symmetry,

$$
(\Psi(x), A(x)) \mapsto(\Psi(x+t), A(x+t)), \quad \forall t \in \mathbb{R}^{2} ;
$$

The rotation and reflection symmetry,

$$
(\Psi(x), A(x)) \mapsto\left(\Psi\left(R^{-1} x\right), R A\left(R^{-1} x\right)\right), \quad \forall R \in O(2) .
$$

2.2 Flux quantization. One can show that under certain boundary conditions (e.g., 'gauge-periodic', see below, or if $\Omega=\mathbb{R}^{2}$ and $\left.\mathcal{E}_{\Omega}<\infty\right)$ the magnetic flux through $\Omega$ is quantized:

$$
\Phi(A):=\int_{\Omega} \operatorname{curl} A=2 \pi n
$$

for some integer $n$.

2.3 Elementary solutions. There are two immediate solutions to the Ginzburg-Landau equations that are homogeneous in $\Psi$. These are the perfect superconductor solution where $\Psi_{S} \equiv 1$ and $A_{S} \equiv 0$, and the normal (or non-superconducting) solution where $\Psi_{N}=0$ and $A_{N}$ is such that $\operatorname{curl} A_{N}=: b$ is constant. (We see that the perfect superconductor is a solution only when $\Phi(A)=0$. On the other hand, there is a normal solution, $\left(\Psi_{N}=0, A_{N}, \operatorname{curl} A_{N}=\right.$ constant), for any condition on $\Phi(A)$.)

Moreover, for any integer $n$ there is a $(n-)$ vortex solution of the form

$$
\Psi^{(n)}(x)=f_{n}(r) e^{i n \theta} \quad \text { and } \quad A^{(n)}(x)=a_{n}(r) \nabla(n \theta),
$$

where $(r, \theta)$ are the polar coordinates of $x \in \mathbb{R}^{2}$, unique up to symmetry transformations (see $[9,17]$ ). Note that $\Phi\left(A^{(n)}\right)=n$. 


\section{Lattice states}

3.1 Periodicity. Our focus in this paper is on states $(\Psi, A)$ defined on all of $\mathbb{R}^{2}$, but whose physical properties, the density of superconducting pairs of electrons, $n_{s}:=|\Psi|^{2}$, the magnetic field, $B:=\operatorname{curl} A$, and the current density, $J:=\operatorname{Im}\left(\bar{\Psi} \nabla_{A} \Psi\right)$, are doubly-periodic with respect to some lattice $\mathcal{L}$. We call such states $\mathcal{L}$-lattice states.

One can show that a state $(\Psi, A) \in H_{\text {loc }}^{1}\left(\mathbb{R}^{2} ; \mathbb{C}\right) \times H_{\text {loc }}^{1}\left(\mathbb{R}^{2} ; \mathbb{R}^{2}\right)$ is a $\mathcal{L}$-lattice state if and only if translation by an element of the lattice results in a gauge transformation of the state, that is, for each $t \in \mathcal{L}$, there exists a function $g_{t} \in H_{\text {loc }}^{2}\left(\mathbb{R}^{2} ; \mathbb{R}\right)$ such that

$$
\Psi(x+t)=e^{i g_{t}(x)} \Psi(x) \text { and } A(x+t)=A(x)+\nabla g_{t}(x)
$$

almost everywhere.

It is clear that the gauge, translation, and rotation symmetries of the Ginzburg-Landau equations map lattice states to lattice states. In the case of the gauge and translation symmetries, the lattice with respect to which the solution is periodic does not change, whereas with the rotation symmetry, the lattice is rotated as well. It is a simple calculation to verify that the magnetic flux per cell of solutions is also preserved under the action of these symmetries.

Note that $(\Psi, A)$ is defined by its restriction to a single cell and can be reconstructed from this restriction by lattice translations.

3.2 Flux quantization. The important property of lattice states is that the magnetic flux through a lattice cell is quantized, i.e. (2.4) holds, with $\Omega$ any fundamental cell of the lattice.

Indeed, if $|\Psi|>0$ on the boundary of the cell, we can write $\Psi=|\Psi| e^{i \theta}$ and $0 \leq \theta<2 \pi$. The periodicity of $n_{s}$ and $J$ ensure the periodicity of $\nabla \theta-A$ and therefore by Green's theorem, $\int_{\Omega} \operatorname{curl} A=\oint_{\partial \Omega} A=$ $\oint_{\partial \Omega} \nabla \theta$ and this function is equal to $2 \pi n$ since $\Psi$ is single-valued.

Equation (2.4) then imposes a condition on the area of a cell, namely, (1.3).

3.3 Lattice shape. In order to define the shape of a lattice, we identify $x \in \mathbb{R}^{2}$ with $z=x_{1}+i x_{2} \in \mathbb{C}$, and view $\mathcal{L}$ as a subset of $\mathbb{C}$. It is a well-known fact (see [4]) that any lattice $\mathcal{L} \subseteq \mathbb{C}$ can be given a basis $r, r^{\prime}$ such that the ratio $\tau=\frac{r^{\prime}}{r}$ satisfies the inequalities:

(i) $|\tau| \geq 1$

(ii) $\operatorname{Im} \tau>0$;

(iii) $-\frac{1}{2}<\operatorname{Re} \tau \leq \frac{1}{2}$, and $\operatorname{Re} \tau \geq 0$ if $|\tau|=1$.

Although the basis is not unique, the value of $\tau$ is, and we will use that as a measure of the shape of the lattice.

Using the rotation symmetry we can assume that $\mathcal{L}$ has as a basis $\left\{r e_{1}, r \tau\right\}$, where $r$ is a positive real number and $e_{1}=(1,0)$.

\section{Fixing the gauge and rescaling}

In this section we fix the gauge for solutions, $(\Psi, A)$, of $(1.2)$ and then rescale them to eliminate the dependence of the size of the lattice on $b$. Our space will then depend only on the number of quanta of flux and the shape of the lattice.

4.1 Fixing the gauge. The gauge symmetry allows one to fix solutions to be of a desired form. Let $A_{0}^{b}(x)=\frac{b}{2} J x$, where $J$ is the symplectic matrix

$$
J=\left(\begin{array}{cc}
0 & -1 \\
1 & 0
\end{array}\right)
$$

We will use the following preposition, first used by [24] and proved in [28] (we provide an alternate proof in Appendix A). 
Proposition 4.1. Let $(\Psi, A)$ be an $\mathcal{L}$-lattice state, and let $b$ be the average magnetic flux per cell. Then there is a $\mathcal{L}$-lattice state $\left(\phi, A_{0}^{b}+\alpha\right)$, that is gauge-equivalent to a translation of $(\Psi, A)$, such that

(i) $\phi(x+t)=e^{\frac{i b}{2} x \cdot J t} \phi(x)$ and $\alpha(x+t)=\alpha(x)$ for all $t$ in a fixed basis of $\mathcal{L}$;

(ii) $\alpha$ has mean zero: $\int_{\Omega} \alpha=0$;

(iii) $\alpha$ is divergence-free: $\operatorname{div} \alpha=0$.

4.2 Rescaling. Suppose, that we have a $\mathcal{L}$-lattice state $(\Psi, A)$, where $\mathcal{L}$ has shape $\tau$. Now let $b$ be the average magnetic flux per cell of the state and $n$ the quanta of flux per cell. From the quantization of the flux, we know that

$$
b=\frac{2 \pi n}{|\Omega|}=\frac{\left(r^{\tau}\right)^{2}}{r^{2}} n, \quad r^{\tau}:=\left(\frac{2 \pi}{\operatorname{Im} \tau}\right)^{\frac{1}{2}},
$$

We set $\sigma:=\left(\frac{n}{b}\right)^{\frac{1}{2}}=\frac{r}{r^{\tau}}$. We now define the rescaled fields $(\psi, a)$ to be

$$
(\psi(x), a(x)):=(\sigma \Psi(\sigma x), \sigma A(\sigma x)) .
$$

Let $\mathcal{L}^{\tau}$ be the lattice spanned by $r^{\tau}$ and $r^{\tau} \tau$, with $\Omega^{\tau}$ being a primitive cell of that lattice. We note that $\left|\Omega^{\tau}\right|=2 \pi$. We summarize the effects of the rescaling above:

(A) $(\psi, a)$ is a $\mathcal{L}^{\tau}$-lattice state.

(B) $\frac{1}{|\Omega|} E_{\Omega}(\Psi, A)=\mathcal{E}_{\lambda}(\psi, a)$, where $\lambda=\frac{\kappa^{2} n}{b}$ and

$$
\mathcal{E}_{\lambda}(\psi, \alpha)=\frac{\kappa^{4}}{\left|\Omega^{\tau}\right| \lambda^{2}} \int_{\Omega^{\tau}}\left(\left|\nabla_{a} \psi\right|^{2}+|\operatorname{curl} a|^{2}+\frac{\kappa^{2}}{2}\left(|\psi|^{2}-\frac{\lambda}{\kappa^{2}}\right)^{2}\right) d x .
$$

(C) $\Psi$ and $A$ solve the Ginzburg-Landau equations if and only if $\psi$ and $a$ solve

$$
\begin{gathered}
\left(-\Delta_{a}-\lambda\right) \psi=-\kappa^{2}|\psi|^{2} \psi, \\
\text { curl }^{*} \operatorname{curl} a=\operatorname{Im}\left(\bar{\psi} \nabla_{a} \psi\right)
\end{gathered}
$$

for $\lambda=\frac{\kappa^{2} n}{b}$. The latter equations are valid on $\Omega^{\tau}$ with the boundary conditions given in the next statement.

(D) If $(\Psi, A)$ is of the form described in Proposition 4.1, then $\psi$ and $a$ satisfy

(a) $\psi(x+t)=e^{\frac{i n}{2} x \cdot J t} \psi(x)$ and $\alpha(x+t)=\alpha(x)$, for $t=r^{\tau}, r^{\tau} \tau$, where $a=A_{0}^{n}+\alpha$, with $A_{0}^{n}(x):=$ (b) $\int_{\Omega^{\tau}}^{\frac{n}{2} J x}=0$

(c) $\operatorname{div} \alpha=0$.

Our problem then is, for each $n=1,2, \ldots$, find $(\psi, a)$, solving the rescaled Ginzburg-Landau equations (4.3) and satisfying (i), and among these find the one that minimizes the average energy $\mathcal{E}_{\lambda}$.

\section{Asymptotics of solutions to (4.3)}

In this section we derive properties of families of solutions of (4.3) depending on $b$, in the regime of $b \rightarrow \kappa^{2}$, provided such families exist. It might be convenient to reparametrize such families assuming that $b$, or $\lambda$, depends on a parameter $\epsilon \rightarrow 0$. Most of the results of this section were first stated in [1] (see also [10]). In what follows we use the notation

$$
\langle f\rangle:=\frac{1}{\left|\Omega^{\tau}\right|} \int_{\Omega^{\tau}} f
$$

for the average of a function $f$ over the lattice cell $\Omega^{\tau}$. The main result of this section is the following 
Proposition 5.1. Assume the equations (4.3) have a family, $\left(\psi_{\epsilon}, a_{\epsilon}, \lambda_{\epsilon}\right), \epsilon \rightarrow 0$, of Abrikosov lattice solutions, with $\left\langle\operatorname{curl} a_{\epsilon}\right\rangle=n$, satisfying

$$
\psi_{\epsilon}=\epsilon \psi_{0}+O\left(\epsilon^{3}\right), a_{\epsilon}=A_{0}^{n}+\epsilon^{2} a_{1}+O\left(\epsilon^{4}\right), \lambda_{\epsilon}=n+\epsilon^{2} \lambda_{1}+O\left(\epsilon^{4}\right),
$$

(with the first and second derivatives of the remainders obeying similar estimates). Then $\psi_{0}, a_{1}$ and $\lambda_{1}$ satisfy the equations

$$
-\Delta_{A_{0}^{n}} \psi_{0}=n \psi_{0}, \text { and } \operatorname{curl} a_{1}=\frac{1}{2}\left\langle\left|\psi_{0}\right|^{2}\right\rangle-\frac{1}{2}\left|\psi_{0}\right|^{2},
$$

and

$$
\lambda_{1}=\left[\frac{1}{2}+\left(\kappa^{2}-\frac{1}{2}\right) \beta\left(\psi_{0}\right)\right]\left\langle\left|\psi_{0}\right|^{2}\right\rangle
$$

where $\beta\left(\psi_{0}\right)$ is the Abrikosov parameter, defined by

$$
\beta\left(\psi_{0}\right):=\frac{\left\langle\left|\psi_{0}\right|^{4}\right\rangle}{\left\langle\left|\psi_{0}\right|^{2}\right\rangle^{2}}
$$

Furthermore, the energy is expressed as

$$
\mathcal{E}_{\lambda_{\epsilon}}\left(\psi_{\epsilon}, a_{\epsilon}\right)=\frac{\kappa^{2}}{2}+\frac{n^{2} \kappa^{4}}{\lambda_{\epsilon}^{2}}-\frac{\kappa^{4} \epsilon^{4}}{2 n^{2}}\left[\left(\kappa^{2}-\frac{1}{2}\right) \beta\left(\psi_{0}\right)+\frac{1}{2}\right]\left\langle\left|\psi_{0}\right|^{2}\right\rangle^{2}+O\left(\epsilon^{6}\right) .
$$

Proof. Plugging (5.1) into (4.3) and taking $\epsilon \rightarrow 0$ gives the first equation in (5.2) and

$$
\operatorname{curl}^{*} \operatorname{curl} a_{1}=\operatorname{Im}\left(\bar{\psi}_{0} \nabla_{A_{0}^{n}} \psi_{0}\right) .
$$

We show now that

$$
\operatorname{Im}\left(\bar{\psi}_{0} \nabla_{A_{0}^{n}} \psi_{0}\right)=-\frac{1}{2} \operatorname{curl}^{*}\left|\psi_{0}\right|^{2} .
$$

(Recall, that for a scalar function, $f(x) \in \mathbb{R}$, $\operatorname{curl}^{*} f=\left(\partial_{2} f,-\partial_{1} f\right)$ is a vector.) It follows from (6.3), Section 6 , that $\psi_{0}$ satisfies the first order equation

$$
\left(\left(\nabla_{A_{0}^{n}}\right)_{1}+i\left(\nabla_{A_{0}^{n}}\right)_{2}\right) \psi_{0}=0 .
$$

Multiplying this relation by $\bar{\psi}_{0}$, we obtain $\bar{\psi}_{0}\left(\nabla_{A_{0}^{n}}\right)_{1} \psi_{0}+i \bar{\psi}_{0}\left(\nabla_{A_{0}^{n}}\right)_{2} \psi_{0}=0$. Taking imaginary and real parts of this equation gives

$$
\operatorname{Im} \bar{\psi}_{0}\left(\nabla_{A_{0}^{n}}\right)_{1} \psi_{0}=-\operatorname{Re} \bar{\psi}_{0}\left(\nabla_{A_{0}^{n}}\right)_{2} \psi_{0}=-\frac{1}{2} \partial_{x_{2}}\left|\psi_{0}\right|^{2}
$$

and

which, in turn, gives (5.7).

$$
\operatorname{Im} \bar{\psi}_{0}\left(\nabla_{A_{0}^{n}}\right)_{2} \psi_{0}=\operatorname{Re} \bar{\psi}_{0}\left(\nabla_{A_{0}^{n}}\right)_{1} \psi_{0}=\frac{1}{2} \partial_{x_{1}}\left|\psi_{0}\right|^{2},
$$

The equations (5.6) and (5.7) give $\operatorname{curl} a_{1}=H-\frac{1}{2}\left|\psi_{0}\right|^{2}$, with $H$ a constant of integration. $H$ has to be chosen so that $\int_{\Omega^{\tau}} \operatorname{curl} a_{1}=0$, which gives the second equation in (5.2).

Now we prove (5.3). We take the inner product of (4.3a) with $\psi_{0}$, use that the operator $-\Delta_{a}$ is self-adjoint and $\left(-\Delta_{a}-n\right) \psi_{0}=0$, substitute the expansions (5.1) and take $\epsilon=0$, to obtain

$$
-\lambda_{1} \int_{\Omega^{\tau}}\left|\psi_{0}\right|^{2}+2 i \int_{\Omega^{\tau}} \bar{\psi}_{0} a_{1} \cdot \nabla_{A_{0}^{n}} \psi_{0}+\kappa^{2} \int_{\Omega^{\tau}}\left|\psi_{0}\right|^{4}=0
$$

This expression implies that the imaginary part of the second term on the left hand side of (5.9) is zero. (We arrive at the same conclusion by integrating by parts and using that $\operatorname{div} a_{1}=0$.) Therefore

$$
2 i \int_{\Omega^{\tau}} \bar{\psi}_{0} a_{1} \cdot \nabla_{A_{0}^{n}} \psi_{0}=-2 \int_{\Omega^{\tau}} a_{1} \cdot \operatorname{Im}\left(\bar{\psi}_{0} \nabla_{A_{0}^{n}} \psi_{0}\right)=-2 \int_{\Omega^{\tau}} a_{1} \cdot \operatorname{curl}^{*} \operatorname{curl} a_{1} \cdot
$$


Integrating the last term by parts, we obtain $2 i \int_{\Omega^{\tau}} \bar{\psi}_{0} a_{1} \cdot \nabla_{A_{0}^{n}} \psi_{0}=-2 \int_{\Omega^{\tau}}\left(\operatorname{curl} a_{1}\right)^{2}$. Using this equation and the second equation in (5.2), we obtain

$$
2 i \int_{\Omega^{\tau}} \bar{\psi}_{0} a_{1} \cdot \nabla_{A_{0}^{n}} \psi_{0}=-\frac{1}{2} \int_{\Omega^{\tau}}\left|\psi_{0}\right|^{4}+\frac{1}{2}\left\langle\left|\psi_{0}\right|^{2}\right\rangle \int_{\Omega^{\tau}}\left|\psi_{0}\right|^{2}
$$

This equation together with (5.9) and the definition (5.4) gives (5.3).

Now, we prove statement (5.5) about the Ginzburg-Landau energy. Taking the inner product of (4.3a) with $\psi$ and integrating by parts gives

$$
\int_{\Omega^{\tau}}\left|\nabla_{a} \psi\right|^{2}=\int_{\Omega^{\tau}}\left(\lambda|\psi|^{2}-\kappa^{2}|\psi|^{4}\right)
$$

Substituting this into the expression for the energy, we find

$$
\mathcal{E}_{\lambda}(\psi, a)=\frac{\kappa^{4}}{\lambda^{2}}\left(\frac{\lambda^{2}}{2 \kappa^{2}}-\frac{\kappa^{2}}{2}\left\langle|\psi|^{4}\right\rangle+\left\langle|\operatorname{curl} a|^{2}\right\rangle\right)
$$

Using the expansions (5.1) and the facts that $\operatorname{curl} A_{0}^{n}=n$ and $\left\langle\operatorname{curl} a_{1}\right\rangle=0$ gives

$$
\mathcal{E}_{\lambda_{\epsilon}}\left(\psi_{\epsilon}, a_{\epsilon}\right)=\frac{\kappa^{2}}{2}+\frac{n^{2} \kappa^{4}}{\lambda_{\epsilon}^{2}}+\frac{\kappa^{4} \epsilon^{4}}{\lambda_{\epsilon}^{2}}\left(-\frac{\kappa^{2}}{2}\left\langle\left|\psi_{0}\right|^{4}\right\rangle+\left\langle\left|\operatorname{curl} a_{1}\right|^{2}\right\rangle\right)+O\left(\epsilon^{6}\right) .
$$

Next, using the second equation in (5.2) together with the last equation in (5.1) and the definition (5.4) yields (5.5).

Eqn (5.3) fixes the parameter $\epsilon$ uniquely up to the normalization of $\psi_{0}$. Indeed, we observe that the third equation in (5.1) implies $\epsilon^{2}=\frac{\lambda-n}{\lambda_{1}}+O\left((\lambda-n)^{2}\right)$, which, together with the definition $\lambda=\frac{\kappa^{2} n}{b}$ and (5.3), yields

$$
\epsilon^{2}=\frac{n\left(\kappa^{2}-b\right)}{\kappa^{2}\left[\left(\kappa^{2}-\frac{1}{2}\right) \beta\left(\psi_{0}\right)+\frac{1}{2}\right]\left\langle\left|\psi_{0}\right|^{2}\right\rangle}+O\left(\left(\kappa^{2}-b\right)^{2}\right)
$$

This equation implies the following necessary condition on existence of the solutions:

$$
b \leq \kappa^{2} \text { if }\left(\kappa^{2}-\frac{1}{2}\right) \beta\left(\psi_{0}\right)+\frac{1}{2} \geq 0 \quad \text { and } \quad b>\kappa^{2} \text { if }\left(\kappa^{2}-\frac{1}{2}\right) \beta\left(\psi_{0}\right)+\frac{1}{2}<0 .
$$

(5.13) together with (5.5) yields

$$
\mathcal{E}_{\lambda_{\epsilon}}\left(\psi_{\epsilon}, a_{\epsilon}\right)=\frac{\kappa^{2}}{2}+b-\frac{\left(\kappa^{2}-b\right)^{2}}{\left(2 \kappa^{2}-1\right) \beta\left(\psi_{0}\right)+1}+O\left(\left(\kappa^{2}-b\right)^{3}\right)
$$

\section{The linear problem}

In this section we solve the linear problem: $-\Delta_{A_{0}^{n}} \psi_{0}=n \psi_{0}$, for $\psi_{0}$ satisfying the gauge - periodic boundary condition $\psi_{0}(x+t)=e^{\frac{i n}{2} x \cdot J t} \psi_{0}(x)$, for $t=r^{\tau}, r^{\tau} \tau$, (see (5.2) and (D).) We introduce the harmonic oscillator annihilation and creation operators, $\alpha^{n}$ and $\left(\alpha^{n}\right)^{*}$, with

$$
\alpha^{n}:=\left(\nabla_{A_{0}^{n}}\right)_{1}+i\left(\nabla_{A_{0}^{n}}\right)_{2}=\partial_{x_{1}}+i \partial_{x_{2}}+\frac{n}{2} x_{1}+\frac{i n}{2} x_{2} .
$$

One can verify that these operators satisfy the following relations:

1. $\left[\alpha^{n},\left(\alpha^{n}\right)^{*}\right]=2 \operatorname{curl} A_{0}^{n}=2 n$;

2. $-\Delta_{A_{0}^{n}}-n=\left(\alpha^{n}\right)^{*} \alpha^{n}$. 
As for the harmonic oscillator (see for example [18]), this gives explicit information about $\sigma\left(-\Delta_{A_{0}^{n}}\right)$ :

$$
\sigma\left(-\Delta_{A_{0}^{n}}\right)=\{(2 k+1) n: k=0,1,2, \ldots\}
$$

and each eigenvalue is of the same multiplicity. Furthermore, the above properties imply

$$
\operatorname{null}\left(-\Delta_{A_{0}^{n}}-n\right)=\operatorname{null} \alpha^{n} .
$$

We can now prove the following.

Proposition 6.1. null $\left(-\Delta_{A_{0}^{n}}-n\right)$ is given by

$$
\operatorname{null}\left(-\Delta_{A_{0}^{n}}-n\right)=\left\{e^{\frac{i n}{2} x_{2}\left(x_{1}+i x_{2}\right)} \sum_{k=-\infty}^{\infty} c_{k} e^{i k \sqrt{2 \pi \operatorname{Im} \tau}\left(x_{1}+i x_{2}\right)} \mid c_{k+n}=e^{i n \pi \tau} e^{i 2 k \pi \tau} c_{k}\right\}
$$

and therefore, in particular, $\operatorname{dim}_{\mathbb{C}} \operatorname{null}\left(-\Delta_{A_{0}^{n}}-n\right)=n$.

Proof. We find null $\alpha^{n}$. A simple calculation gives the following operator equation

$$
e^{\frac{n}{4}|x|^{2}} \alpha^{n} e^{-\frac{n}{4}|x|^{2}}=\partial_{x_{1}}+i \partial_{x_{2}} .
$$

This immediately proves that $\psi \in$ null $\alpha^{n}$ if and only if $\xi=e^{\frac{n}{4}|x|^{2}} \psi$ satisfies $\partial_{x_{1}} \xi+i \partial_{x_{2}} \xi=0$. We now identify $x \in \mathbb{R}^{2}$ with $z=x_{1}+i x_{2} \in \mathbb{C}$ and see that this means that $\xi$ is analytic. We therefore define the entire function $\Theta$ to be

$$
\Theta(z)=e^{-\frac{n z^{2}}{2 \pi \operatorname{Im} \tau}} \xi\left(\sqrt{\frac{2}{\pi \operatorname{Im} \tau}} z\right)=e^{\frac{n\left(|z|^{2}-z^{2}\right)}{2 \pi \operatorname{Im} \tau}} \psi\left(\sqrt{\frac{2}{\pi \operatorname{Im} \tau} z}\right) .
$$

The quasiperiodicity of $\psi$ transfers to $\Theta$ as follows

$$
\begin{gathered}
\Theta(z+\pi)=\Theta(z), \\
\Theta(z+\pi \tau)=e^{-2 i n z} e^{-i n \pi \tau} \Theta(z) .
\end{gathered}
$$

To complete the proof, we now need to show that the space of the analytic functions which satisfy these relations form a vector space of dimension $n$. It is easy to verify that the first relation ensures that $\Theta$ have a absolutely convergent Fourier expansion of the form

$$
\Theta(z)=\sum_{k=-\infty}^{\infty} c_{k} e^{2 k i z}
$$

The second relation, on the other hand, leads to relation for the coefficients of the expansion. Namely, we have $c_{k+n}=e^{i n \pi \tau} e^{2 k i \pi \tau} c_{k}$ and that means such functions are determined solely by the values of $c_{0}, \ldots, c_{n-1}$ and therefore form an $n$-dimensional vector space.

\section{Reformulation of the problem}

In this section we reduce two equations (4.3) for $\psi$ and $a$ to a single equation for $\psi$. We introduce the spaces $\mathscr{L}_{n}(\tau):=L^{2}\left(\Omega^{\tau}, \mathbb{C}\right)$ and $\mathscr{L}(\tau):=\left\{a \in L^{2}\left(\Omega^{\tau}, \mathbb{R}^{2}\right) \mid\langle a\rangle=0\right.$, div $a=0$, in the distributional sense\}, with the domains being the Sobolev space of order two, $\mathscr{H}_{n}(\tau)$, whose elements satisfy the quasiperiodic boundary condition (a) in (D) above, and the Sobolev space of order two, $\mathscr{H}(\tau)$, whose elements satisfy the periodic boundary conditions with respect to $\mathcal{L}^{\tau}$, have mean zero, and are divergence free. Substituting $a=A_{0}^{n}+\alpha$, we rewrite (4.3) as

$$
\left(L^{n}-\lambda\right) \psi+2 i \alpha \cdot \nabla_{A_{0}^{n}} \psi+|\alpha|^{2} \psi+\kappa^{2}|\psi|^{2} \psi=0,
$$




$$
\left(M+|\psi|^{2}\right) \alpha-\operatorname{Im}\left(\bar{\psi} \nabla_{A_{0}^{n}} \psi\right)=0
$$

where

$$
L^{n}:=-\Delta_{A_{0}^{n}} \text { and } M:=\operatorname{curl}^{*} \text { curl, }
$$

defined on the spaces $\mathscr{L}_{n}(\tau)$ and $\mathscr{L}(\tau)$. Their properties that will be used below are summarized in the following propositions:

Proposition 7.1. $L^{n}$ is a self-adjoint operator on $\mathscr{H}_{n}(\tau)$ with spectrum $\sigma\left(L^{n}\right)=\{(2 k+1) n: k=$ $0,1,2, \ldots\}$ and $\operatorname{dim}_{\mathbb{C}} \operatorname{null}\left(L^{n}-n\right)=n$.

Proposition 7.2. $M$ is a strictly positive operator on $\mathscr{H}(\tau)$ with discrete spectrum.

The proofs of these results are standard and, for the convenience of the reader, are given below.

Proof of Proposition 7.2. The fact that $M$ is positive follows immediately from its definition. We note that its being strictly positive is the result of restricting its domain to elements having mean zero.

Proof of Proposition 7.1. First, we note that $L^{n}$ is clearly a positive self-adjoint operator. To see that it has discrete spectrum, we first note that the inclusion $H^{2} \hookrightarrow L^{2}$ is compact for bounded domains in $\mathbb{R}^{2}$ with Lipschitz boundary (which certainly includes lattice cells). Then for any $z$ in the resolvent set of $L^{n},\left(L^{n}-z\right)^{-1}: L^{2} \rightarrow H^{2}$ is bounded and therefore $\left(L^{n}-z\right)^{-1}: L^{2} \rightarrow L^{2}$ is compact. In fact, the spectrum of $L^{n}$ was found explicitly in the previous section. This completes the proof of Proposition 7.1.

We first solve the second equation (7.1b) for $\alpha$ in terms of $\psi$. By the gauge invariance we have to solve the equations $\left(M+|\psi|^{2}\right) \alpha-\operatorname{Im}\left(\bar{\psi} \nabla_{A_{0}^{n}+\nabla \chi+h} \psi\right)=0, \operatorname{div} \operatorname{Im}\left(\bar{\psi} \nabla_{A_{0}^{n}+\alpha+\nabla \chi+h} \psi\right)=0$ and $\left\langle\operatorname{Im}\left(\bar{\psi} \nabla_{A_{0}^{n}+\alpha+\nabla \chi+h} \psi\right)\right\rangle=0$ for $\alpha \in \mathscr{H}(\tau), \chi$ periodic, and $h \in \mathbb{R}^{2}$. This is done using the implicit function theorem. It is convenient to solve first the last two equations for $\chi$, periodic and $h \in \mathbb{R}^{2}$, and then the first equation for $\alpha \in \mathscr{H}(\tau)$. We omit the details. Absorbing the gauge $\chi+h \cdot x$ into the solution, we write the solution $\alpha$ as $\alpha=\alpha(\psi)$, where

$$
\alpha(\psi)=\left(M+|\psi|^{2}\right)^{-1} \operatorname{Im}\left(\bar{\psi} \nabla_{A_{0}^{n}} \psi\right) .
$$

We collect the elementary properties of the map $\alpha$ in the following proposition, where we identify $\mathscr{H}_{n}(\tau)$ with a real Banach space using $\psi \leftrightarrow \vec{\psi}:=(\operatorname{Re} \psi, \operatorname{Im} \psi)$.

Proposition 7.3. The unique solution, $\alpha(\psi)$, of $(7.1 \mathrm{~b})$ maps $\mathscr{H}_{n}(\tau)$ to $\mathscr{H}(\tau)$ and has the following properties:

(a) $\alpha(\cdot)$ is analytic as a map between real Banach spaces.

(b) $\alpha(0)=0$.

(c) For any $\delta \in \mathbb{R}, \alpha\left(e^{i \delta} \psi\right)=\alpha(\psi)$.

Proof. The only statement that does not follow immediately from the definition of $\alpha$ is (a). It is clear that $\operatorname{Im}\left(\bar{\psi} \nabla_{A_{0}^{n}} \psi\right)$ is real-analytic as it is a polynomial in $\psi$ and $\nabla \psi$, and their complex conjugates. We also note that $(M-z)^{-1}$ is complex-analytic in $z$ on the resolvent set of $M$, and therefore, $\left(M+|\psi|^{2}\right)^{-1}$ is analytic. (a) now follows.

Now we substitute the expression (7.3) for $\alpha$ into (7.1a) to get a single equation

$$
F(\lambda, \psi)=0,
$$

where the map $F: \mathbb{R} \times \mathscr{H}_{n}(\tau) \rightarrow \mathscr{L}_{n}(\tau)$ is defined as

$$
F(\lambda, \psi)=\left(L^{n}-\lambda\right) \psi+2 i \alpha(\psi) \cdot \nabla_{A_{0}^{n}} \psi+|\alpha(\psi)|^{2} \psi+\kappa^{2}|\psi|^{2} \psi .
$$

For a map $F(\psi)$, we denote by $\partial_{\psi} F(\phi)$ its Gâteaux derivative in $\psi$ at $\phi$. The following proposition lists some properties of $F$. 


\section{Proposition 7.4.}

(a) $F$ is analytic as a map between real Banach spaces,

(b) for all $\lambda, F(\lambda, 0)=0$,

(c) for all $\lambda, \partial_{\psi} F(\lambda, 0)=L^{n}-\lambda$,

(d) for all $\delta \in \mathbb{R}, F\left(\lambda, e^{i \delta} \psi\right)=e^{i \delta} F(\lambda, \psi)$.

(e) for all $\psi,\langle\psi, F(\lambda, \psi)\rangle \in \mathbb{R}$.

Proof. The first property follows from the definition of $F$ and the corresponding analyticity of $\alpha(\psi)$. (b) through (d) are straightforward calculations. For (e), we calculate that

$$
\begin{aligned}
\langle\psi, F(\lambda, \psi)\rangle & =\left\langle\psi,\left(L^{n}-\lambda\right) \psi\right\rangle+2 i \int_{\Omega^{\tau}} \bar{\psi} \alpha(\psi) \cdot \nabla \psi \\
& +2 \int_{\Omega^{\tau}}\left(\alpha(\psi) \cdot A_{0}^{n}\right)|\psi|^{2}+\int_{\Omega^{\tau}}|\alpha(\psi)|^{2}|\psi|^{2}+\kappa^{2} \int_{\Omega^{\tau}}|\psi|^{4} .
\end{aligned}
$$

The final three terms are clearly real and so is the first because $L^{n}-\lambda$ is self-adjoint. For the second term we calculate the complex conjugate and see that

$$
\overline{2 i \int_{\Omega^{\tau}} \bar{\psi} \alpha(\psi) \cdot \nabla \psi}=-2 i \int_{\Omega^{\tau}} \psi \alpha(\psi) \cdot \nabla \bar{\psi}=2 i \int_{\Omega^{\tau}}(\nabla \psi \cdot \alpha(\psi)) \bar{\psi},
$$

where we have integrated by parts and used the fact that the boundary terms vanish due to the periodicity of the integrand and that $\operatorname{div} \alpha(\psi)=0$. Thus this term is also real and (e) is established.

\section{Reduction to a finite-dimensional problem}

In this section we reduce the problem of solving the equation $F(\lambda, \psi)=0$ to a finite dimensional problem. We address the latter in the next section. We use the standard method of Lyapunov-Schmidt reduction. Let $X:=\mathscr{H}_{n}(\tau)$ and $Y:=\mathscr{L}_{n}(\tau)$ and let $K=\operatorname{null}\left(L^{n}-n\right)$. We let $P$ be the Riesz projection onto $K$, that is,

$$
P:=-\frac{1}{2 \pi i} \oint_{\gamma}\left(L^{n}-z\right)^{-1} d z
$$

where $\gamma \subseteq \mathbb{C}$ is a contour around $n$ that contains no other points of the spectrum of $L^{n}$. This is possible since $n$ is an isolated eigenvalue of $L^{n}$. $P$ is a bounded, orthogonal projection, and if we let $Z:=$ null $P$, then $Y=K \oplus Z$. We also let $Q:=I-P$, and so $Q$ is a projection onto $Z$.

The equation $F(\lambda, \psi)=0$ is therefore equivalent to the pair of equations

$$
\begin{aligned}
& P F(\lambda, P \psi+Q \psi)=0, \\
& Q F(\lambda, P \psi+Q \psi)=0 .
\end{aligned}
$$

We will now solve (8.3) for $w=Q \psi$ in terms of $\lambda$ and $v=P \psi$. To do this, we introduce the map $G: \mathbb{R} \times K \times Z \rightarrow Z$ to be $G(\lambda, v, w):=Q F(\lambda, v+w)$. Applying the Implicit Function Theorem to $G$, we obtain a function $w: \mathbb{R} \times K \rightarrow Z$, defined on a neighbourhood of $(n, 0)$, such that $w=w(\lambda, v)$ is a unique solution to $G(\lambda, v, w)=0$, for $(\lambda, v)$ in that neighbourhood. This solution has the following properties

$$
\begin{gathered}
w(\lambda, v) \text { real-analytic in }(\lambda, v) \\
\|w\|=O\left(\|v\|^{3}\right) \text { and }\left\|\partial_{\lambda} w\right\|=O\left(\|v\|^{3}\right) .
\end{gathered}
$$

The last property follows from (7.3) and (7.5) together with (8.3).

We substitute the solution $w=w(\lambda, v)$ into (8.2) and see that the latter equation in a neighbourhood of $(n, 0)$ is equivalent to the equation (the bifurcation equation)

$$
\gamma(\lambda, v):=P F(\lambda, v+w(\lambda, v))=0 .
$$


Note that $\gamma: \mathbb{R} \times K \rightarrow K$. We have shown that in a neighbourhood of $(n, 0)$ in $\mathbb{R} \times X,(\lambda, \psi)$ solves $F(\lambda, \psi)=0$ if and only if $(\lambda, v)$, with $v=P \psi$, solves (8.6). Moreover, the solution $\psi$ of $F(\lambda, \psi)=0$ can be reconstructed from the solution $v$ of (8.6) according to the formula

$$
\psi=v+w(\lambda, v) .
$$

Finally we note that $w$ and $\gamma$ inherit the symmetry of the original equation:

Lemma 8.1. For every $\delta \in \mathbb{R}, w\left(\lambda, e^{i \delta} v\right)=e^{i \delta} w(\lambda, v)$ and $\gamma\left(\lambda, e^{i \delta} v\right)=e^{i \delta} \gamma(\lambda, v)$.

Proof. We first check that $w\left(\lambda, e^{i \delta} v\right)=e^{i \delta} w(\lambda, v)$. We note that by definition of $w$,

$$
G\left(\lambda, e^{i \delta} v, w\left(\lambda, e^{i \delta} v\right)\right)=0
$$

but by the symmetry of $F$, we also have $G\left(\lambda, e^{i \delta} v, e^{i \delta} w(\lambda, v)\right)=e^{i \delta} G(\lambda, v, w(\lambda, v))=0$. The uniqueness of $w$ then implies that $w\left(\lambda, e^{i \delta} v\right)=e^{i \delta} w(\lambda, v)$. We can now verify that

$$
\begin{aligned}
& \gamma\left(\lambda, e^{i \delta} v\right)=\operatorname{PF}\left(\lambda, e^{i \delta} v+w\left(\lambda, e^{i \delta} v\right)\right) \\
& \left.=e^{i \delta} \operatorname{PF}(\lambda, v+w(\lambda, v))\right\rangle=e^{i \delta} \gamma(\lambda, v)
\end{aligned}
$$

Solving the bifurcation equation (8.6) is a subtle problem, unless $n=1$. In the latter case, this is done in the next section.

We conclude this section with mentioning an approach to finding solutions to the bifurcation equation (8.6) for any $n$. For a fixed $n$, we define the first reduced energy $E_{\lambda}(\psi):=\mathcal{E}_{\lambda}(\psi, a)$, where $a=A_{0}^{n}+\alpha$, with $A_{0}^{n}(x):=\frac{n}{2} J x$ and $\alpha(\psi)=\left(M+|\psi|^{2}\right)^{-1} \operatorname{Im}\left(\bar{\psi} \nabla_{A_{0}^{n}} \psi\right)$ (see (D) and (7.3)). Critical points of this energy solve the equation $F(\lambda, \psi)=0$.

Next, we introduce the finite dimensional effective Ginzburg-Landau energy

$$
e_{\lambda}(v):=E_{\lambda}(v+w(\lambda, v)) .
$$

It is a straightforward to show that

(i) $e_{\lambda}(v)$ has a critical point $v_{0}$ iff $E_{\lambda}(u)$ has a critical point $u_{0}=v_{0}+w\left(\lambda, v_{0}\right)$;

(ii) Critical points, $v_{0}$, of $e_{\lambda}(v)$ solve the equation (8.6);

(iii) $e_{\lambda}(v)$ is gauge invariant, $e_{\lambda}\left(e^{i \delta} v\right)=e_{\lambda}(v)$.

One can use $e_{\lambda}(v)$ to investigate solutions of the equation (8.6) for any $n$.

\section{Proof of Theorem 1.1}

In this section we look at the case $n=1$, and look for solutions near the trivial solution. For convenience we drop the (super)index $n=1$ from the notation. Recall that $\psi_{0}$ is a non-zero element in the nullspace of the operator $L^{n}-n$ acting on $\mathscr{H}_{n}(\tau)$. Since by Proposition 6.1 , this nullspace is a one-dimensional complex subspace for $n=1$, the Abrikosov function, $\beta\left(\psi_{0}\right)$, defined in (5.4), depends only on $\tau$. Therefore we write $\beta(\tau) \equiv \beta\left(\psi_{0}\right)$, so that

$$
\beta(\tau):=\frac{\left\langle\left|\psi_{0}\right|^{4}\right\rangle}{\left\langle\left|\psi_{0}\right|^{2}\right\rangle^{2}}
$$

We begin with the following result which gives the existence and uniqueness of the Abrikosov lattices.

Theorem 9.1. For every $\tau$ there exist $\epsilon>0$ and a branch, $\left(\lambda_{s}, \psi_{s}, \alpha_{s}\right), s \in[0, \sqrt{\epsilon})$, of nontrivial solutions of the rescaled Ginzburg-Landau equations (4.3), unique modulo the global gauge symmetry (apart from 
the trivial solution $\left.\left(1,0, A_{0}\right)\right)$ in a sufficiently small neighbourhood of $\left(1,0, A_{0}\right)$ in $\mathbb{R} \times \mathscr{H}(\tau) \times \mathscr{H}(\tau)$, and such that

$$
\left\{\begin{array}{l}
\lambda_{s}=1+g_{\lambda}\left(s^{2}\right), \\
\psi_{s}=s \psi_{0}+s g_{\psi}\left(s^{2}\right), \\
a_{s}=A_{0}+g_{a}\left(s^{2}\right),
\end{array}\right.
$$

where $(L-1) \psi_{0}=0, g_{\psi}$ is orthogonal to $\operatorname{null}(L-1), g_{\lambda}:[0, \epsilon) \rightarrow \mathbb{R}, g_{\psi}:[0, \epsilon) \rightarrow \mathscr{H}(\tau)$, and $g_{\alpha}:[0, \epsilon) \rightarrow$ $\mathscr{H}(\tau)$ are real-analytic functions such that $g_{\lambda}(0)=0, g_{\psi}(0)=0, g_{\alpha}(0)=0$ and

$$
g_{\lambda}^{\prime}(0)=\left[\left(\kappa^{2}-\frac{1}{2}\right) \beta(\tau)+\frac{1}{2}\right]\left\langle\left|\psi_{0}\right|^{2}\right\rangle .
$$

Proof. The proof of this theorem is a slight modification of a standard result from bifurcation theory. Our goal is to solve the equation (8.6) for $\lambda$. Since the projection $P$, defined there, is rank one and self-adjoint, we have

$$
P \psi=\frac{1}{\left\|\psi_{0}\right\|^{2}}\left\langle\psi_{0}, \psi\right\rangle \psi_{0}, \text { with } \psi_{0} \in \operatorname{null}(L-1) .
$$

We can therefore view the function $\gamma$ in the bifurcation equation (8.6) as a map $\gamma: \mathbb{R} \times \mathbb{C} \rightarrow \mathbb{C}$, where

$$
\gamma(\lambda, s)=\left\langle\psi_{0}, F\left(\lambda, s \psi_{0}+w\left(\lambda, s \psi_{0}\right)\right)\right\rangle .
$$

We now show that $\gamma(\lambda, s) \in \mathbb{R}$ for $s \in \mathbb{R}$. Since the projection $Q$ is self-adjoint, $Q w(\lambda, v)=w(\lambda, v)$, $w(\lambda, v)$ solves $Q F(\lambda, v+w)=0$ and $v=s \psi_{0}$, we have

$$
\left\langle w\left(\lambda, s \psi_{0}\right), F\left(\lambda, s \psi_{0}+w\left(\lambda, s \psi_{0}\right)\right)\right\rangle=\left\langle w\left(\lambda, s \psi_{0}\right), Q F\left(\lambda, s \psi_{0}+w\left(\lambda, s \psi_{0}\right)\right)\right\rangle=0 .
$$

Therefore, for $s \neq 0$,

$$
\left\langle\psi_{0}, F\left(\lambda, s \psi_{0}+w\left(\lambda, s \psi_{0}\right)\right)\right\rangle=s^{-1}\left\langle s \psi_{0}+w\left(\lambda, s \psi_{0}\right), F\left(\lambda, s \psi_{0}+w\left(\lambda, s \psi_{0}\right)\right)\right\rangle,
$$

and this is real by property (e) of Proposition 7.4. Thus, since by Lemma $8.1, \gamma(\lambda, s)=e^{i \arg s} \gamma(\lambda,|s|)$, it therefore suffices to solve the equation

$$
\gamma_{0}(\lambda, s)=0
$$

for the restriction $\gamma_{0}: \mathbb{R} \times \mathbb{R} \rightarrow \mathbb{R}$ of the function $\gamma$ to $\mathbb{R} \times \mathbb{R}$, i.e., for real $s$. It follows from (8.5) that $w\left(\lambda, s \psi_{0}\right)=O\left(s^{3}\right)$, and therefore (9.6) has the trivial branch of solutions $s \equiv 0$ for all $\lambda$. Hence we factorize $\gamma_{0}(\lambda, s)$ as $\gamma_{0}(\lambda, s)=s \gamma_{1}(\lambda, s)$, i.e., we define the function

$$
\gamma_{1}(\lambda, s):=s^{-1} \gamma_{0}(\lambda, s)
$$

and solve the equation $\gamma_{1}(\lambda, s)=0$. The definition of the function $\gamma_{1}(\lambda, s)$ implies that it has the following properties: $\gamma_{1}(\lambda, s)$ is real-analytic, $\gamma_{1}(\lambda,-s)=\gamma_{1}(\lambda, s), \gamma_{1}(1,0)=0$ and, by (7.5) and (8.5), $\partial_{\lambda} \gamma_{1}(1,0)=-\left\|\psi_{0}\right\|^{2} \neq 0$. Hence by a standard application of the Implicit Function Theorem, there is $\epsilon>0$ and a real-analytic function $\widetilde{\phi}_{\lambda}:(-\sqrt{\epsilon}, \sqrt{\epsilon}) \rightarrow \mathbb{R}$ such that $\widetilde{\phi}_{\lambda}(0)=1$ and $\gamma(\lambda, s)=0$ with $|s|<\sqrt{\epsilon}$ if and only if either $s=0$ or $\lambda=\widetilde{\phi}_{\lambda}(|s|)$.

We also note that because of the symmetry, $\widetilde{\phi}_{\lambda}(-|s|)=\widetilde{\phi}_{\lambda}(|s|), \widetilde{\phi}_{\lambda}$ is an even real-analytic function, and therefore must in fact be a function solely of $s^{2}$. We therefore set $\phi_{\lambda}(s)=\widetilde{\phi}_{\lambda}(\sqrt{s})$ for $s \in[0, \epsilon)$, and so $\phi_{\lambda}$ is real-analytic.

We now define $g_{\psi}:[0, \epsilon) \rightarrow \mathscr{H}(\tau)$ to be

$$
g_{\psi}(s)= \begin{cases}\frac{1}{\sqrt{s}} w\left(\phi_{\lambda}(s), \sqrt{s} \psi_{0}\right) & s \neq 0 \\ 0 & s=0\end{cases}
$$


It is easily check that $g_{\psi}$ is real-analytic and satisfies $s g_{\psi}\left(s^{2}\right)=w\left(\widetilde{\phi}_{\lambda}(s), s \psi_{0}\right)$ for any $s \in[0, \sqrt{\epsilon})$.

Now, we know that there is a neighbourhood of $(1,0)$ in $\mathbb{R} \times \mathscr{H}(\tau)$ such that in this neighbourhood $F(\lambda, \psi)=0$ if and only if $\gamma(\lambda, s)=0$ where $P \psi=s \psi_{0}$. By taking a smaller neighbourhood if necessary, we have proven that $F(\lambda, \psi)=0$ in this neighbourhood if and only if either $s=0$ or $\lambda=\phi_{\lambda}\left(s^{2}\right)$. If $s=0$, we have $\psi=s \psi_{0}+w\left(\widetilde{\phi}_{\lambda}(s), s \psi_{0}\right)=0$, which gives the trivial solution. In the other case, $\psi=s \psi_{0}+w\left(\widetilde{\phi}_{\lambda}(s), s \psi_{0}\right)=s \psi_{0}+s g_{\psi}\left(s^{2}\right)$.

If we now also define $g_{\lambda}(s)=1-\phi_{\lambda}(s)$, then the above gives us a neighbourhood of $(1,0)$ in $\mathbb{R} \times \mathscr{H}(\tau)$ such that the only non-trivial solutions of the equation (7.4) are given by the first two equations in (9.2). We now define the function $\tilde{g}_{a}(s)=\alpha\left(\psi_{s}\right)$, where, recall, $\alpha(\psi)$ is defined in (7.3). This function is realanalytic and satisfies $\tilde{g}_{a}(-s)=\alpha\left(-\psi_{s}\right)=\tilde{g}_{a}(s)$, and therefore is really a function of $s^{2}, g_{a}\left(s^{2}\right)$. Define $a_{s}=A_{0}+g_{a}\left(s^{2}\right)$. Then $\left(\lambda_{s}, \psi_{s}, \alpha_{s}\right), s \in[0, \sqrt{\epsilon})$, solves the rescaled Ginzburg-Landau equations (4.3).

We identify (9.2) with (5.1) of Proposition 5.1, with $\epsilon=s$ and $n=1$. Then (5.3) implies (9.3).

Note that the definition $\lambda=\frac{\kappa^{2}}{b}(n=1)$, the first equation (9.2) and the relation (9.3) imply that for $\left(\kappa^{2}-\frac{1}{2}\right) \beta(\tau)+\frac{1}{2} \geq 0$, the bifurcated solution exists for $b \leq \kappa^{2}$, and for $\left(\kappa^{2}-\frac{1}{2}\right) \beta(\tau)+\frac{1}{2}<0$, it exists for $b>\kappa^{2}$. Thus Theorem 9.1, after rescaling to the original variables, implies (I) - (II) of Theorem 1.1.

Recall that $b=\frac{\kappa^{2}}{\lambda}$. Since the function $\lambda_{s}=1+g_{\lambda}\left(s^{2}\right)$ given in Theorem 9.1, obeys $g_{\lambda}(0)=0$ and $g_{\lambda}^{\prime}(0) \neq 0$, provided $\left(\kappa^{2}-\frac{1}{2}\right) \beta(\tau)+\frac{1}{2} \neq 0$, the function $b=\kappa^{2} \lambda_{s}^{-1}$ can be inverted to obtain $s=s(b)$. We can define the family $\left(\psi_{s(b)}, a_{s(b)}, \lambda_{s(b)}\right)$ of $\mathcal{L}^{\tau}$-periodic solutions of the Ginzburg-Landau equations parameterized by average magnetic flux $b$. Since $s(b)$ is real - analytic in $b$, so are $\psi_{s(b)}, a_{s(b)}, \lambda_{s(b)}$. This proves (III) of Theorem 1.1.

Due to (5.13), we can express the bifurcation parameter $s^{2}$ in terms of $b$ as

$$
s^{2}=\frac{\kappa^{2}-b}{\kappa^{2}\left[\left(\kappa^{2}-\frac{1}{2}\right) \beta(\tau)+\frac{1}{2}\right]\left\langle\left|\psi_{0}\right|^{2}\right\rangle}+O\left(\left(\kappa^{2}-b\right)^{2}\right) .
$$

Furthermore, the equations (5.15) implies that the energy of the state $\left(\psi_{s(b)}, a_{s(b)}, \lambda_{s(b)}\right)$,

$$
E_{b}(\tau):=\mathcal{E}_{\lambda(b)}\left(\psi_{s(b)}, a_{s(b)}\right),
$$

where we display the dependence on $\tau$, coming through the solution $\left(\psi_{s}, a_{s}\right)$, has the following form

$$
E_{b}(\tau)=\frac{\kappa^{2}}{2}+b^{2}-\frac{\left(\kappa^{2}-b\right)^{2}}{\left(2 \kappa^{2}-1\right) \beta(\tau)+1}+O\left(\left(\kappa^{2}-b\right)^{3}\right) .
$$

The next result addresses the nature of dependence of solution on $\tau$.

Lemma 9.2. $\left(\lambda_{s}, \psi_{s}, a_{s}\right)$ depend smoothly on $\tau_{1}:=\operatorname{Re} \tau$ and $\tau_{2}:=\operatorname{Im} \tau$.

Proof. By the above we can write $\psi_{s}=s \psi_{0}+w\left(\lambda_{s}, s\right)$ and $a_{s}=A_{0}+\alpha\left(w\left(\lambda_{s}, s\right), s\right)$, where $\alpha(w, s)$ is given by

$$
\alpha(w, s)=\left(M+\left|s \psi_{0}+w\right|^{2}\right)^{-1} \operatorname{Im}\left(\left(\overline{s \psi_{0}+w}\right) \nabla_{A_{0}}\left(s \psi_{0}+w\right)\right),
$$

$w(\lambda, s)$ solves the the equation

$$
(\bar{L}-\lambda) w=-Q\left[2 i \alpha(w, s) \cdot \nabla_{A_{0}}\left(s \psi_{0}+w\right)+|\alpha(w, s)|^{2}\left(s \psi_{0}+w\right)+\kappa^{2}\left|\left(s \psi_{0}+w\right)\right|^{2}\left(s \psi_{0}+w\right)\right],
$$

and $\lambda_{s}$ solves the equation $\gamma(\lambda, s)=0$ (see (9.6)). Here $\bar{L}$ is the restriction of $L$ to $\operatorname{Ran} Q$. From their explicit expressions we see that $\psi_{0}, P$ and $Q$ are smooth in $\tau_{i}$ (recall that $\tau_{2}$ is bounded away from zero). Differentiating (9.13) with respect to $\tau_{i}$ and solving the resulting linear equation for $\partial_{\tau_{i}} w_{s}$, it is not hard to convince oneself that $w_{s}$ is differentiable in $\tau_{i}$. Repeating this procedure, one sees that $w_{s}$ is smooth in $\tau_{i}$. Therefore $\psi_{s}$ and $a_{s}$ are smooth in $\tau_{i}$. For the same reason, $\gamma(\lambda, s):=\operatorname{PF}\left(\lambda, s \psi_{0}+w(\lambda, s)\right)$ is smooth in $\tau_{i}$ and therefore so is $\lambda_{s}$. 
Corollary 9.3. $E_{b}(\tau)$ depends smoothly on $\tau_{1}:=\operatorname{Re} \tau$ and $\tau_{2}:=\operatorname{Im} \tau$.

Proof. To get rid of dependence of the domain of integration in (4.2) on $\tau$, we change the variables of integration in (4.2) as $x=m_{\tau} y$, where $m_{\tau}=(\sqrt{\operatorname{Im} \tau})^{-1}\left(\begin{array}{ll}1 & \operatorname{Re} \tau \\ 0 & \operatorname{Im} \tau\end{array}\right)$, which reduces the integral to the one over the unit square cell. Since the rescaled functions,

$$
\left\{\begin{array}{l}
\tilde{\psi}_{s}(x)=\psi_{s}\left(m_{\tau} x\right), \\
\tilde{a}_{s}(x)=m_{\tau}^{t} a_{s}\left(m_{\tau} x\right),
\end{array}\right.
$$

defined on a $\tau$-independent square lattice, are still smooth in $\tau_{1}:=\operatorname{Re} \tau$ and $\tau_{2}:=\operatorname{Im} \tau$, the result follows.

The next result establishes a relation between the minimizers of the energy and Abrikosov function.

Theorem 9.4. In the case $\kappa>\frac{1}{\sqrt{2}}$, the minimizers, $\tau_{b}$, of $\tau \mapsto E_{b}(\tau)$ are related to the minimizer, $\tau_{*}$, of $\beta(\tau)$, as $\tau_{b}-\tau_{*}=O\left(\mu^{1 / 2}\right)$, where $\mu:=\kappa^{2}-b$. In particular, $\tau_{b} \rightarrow \tau_{*}$ as $b \rightarrow \kappa^{2}$.

Proof. To prove the theorem we note that $E_{b}(\tau)$ is of the form $E_{b}(\tau)=e_{0}+e_{1} \mu+e_{2}(\tau) \mu^{2}+O\left(\mu^{3}\right)$. The first two terms are constant in $\tau$, so we consider $\tilde{E}_{b}(\tau)=e_{2}(\tau)+O(\mu)$. $\tau_{b}$ is also the minimizer of $\tau \mapsto \tilde{E}_{b}(\tau)$ and $\tau_{*}$, of $e_{2}(\tau)$. We have the expansions $\tilde{E}_{b}\left(\tau_{*}\right)-\tilde{E}_{b}\left(\tau_{b}\right)=\frac{1}{2} \tilde{E}_{b}^{\prime \prime}\left(\tau_{b}\right)\left(\tau_{*}-\tau_{b}\right)^{2}+O\left(\left(\tau_{*}-\tau_{b}\right)^{3}\right)$ and $\tilde{E}_{b}\left(\tau_{*}\right)-\tilde{E}_{b}\left(\tau_{b}\right)=-\frac{1}{2} e_{2}^{\prime \prime}\left(\tau_{*}\right)\left(\tau_{*}-\tau_{b}\right)^{2}+O\left(\left(\tau_{*}-\tau_{b}\right)^{3}\right)+O(\mu)$, which imply the desired result.

The following result was discovered numerically in the physics literature and proven in [2] using earlier result of [23]:

Theorem 9.5. The function $\beta(\tau)$ has exactly two critical points, $\tau=e^{i \pi / 3}$ and $\tau=e^{i \pi / 2}$. The first is minimum, whereas the second is a maximum.

Theorems 9.4 and 9.5 imply the remaining statement, (IV), of Theorem 1.1.

Remarks. 1) The applied magnetic field is given by $h_{0}=\frac{1}{2} \partial_{b} E_{b}(\tau)$. From (9.11) we have formally

$$
\begin{array}{r}
h_{0}=b+\frac{\kappa^{2}-b}{\left(2 \kappa^{2}-1\right) \beta(\tau)+1}+O\left(\left(\kappa^{2}-b\right)^{2}\right) \\
=b+\frac{\kappa^{2}}{2}\left\langle\left|\psi_{0}\right|^{2}\right\rangle+O\left(\left(\kappa^{2}-b\right)^{2}\right) .
\end{array}
$$

2) The proof of Theorem 9.1 gives in fact the following abstract result.

Theorem 9.6. Let $X$ and $Y$ be complex Hilbert spaces, with $X$ a dense subset of $Y$, and consider a map $F: \mathbb{R} \times X \rightarrow Y$ that is analytic as a map between real Banach spaces. Suppose that for some $\lambda_{0} \in \mathbb{R}$, the following conditions are satisfied:

(1) $F(\lambda, 0)=0$ for all $\lambda \in \mathbb{R}$,

(2) $\partial_{\psi} F\left(\lambda_{0}, 0\right)$ is self-adjoint and has an isolated eigenvalue at 0 of (geometric) multiplicity 1 ,

(3) For non-zero $\psi_{0} \in$ null $\partial_{\psi} F\left(\lambda_{0}, 0\right),\left\langle\psi_{0}, \partial_{\lambda, \psi} F\left(\lambda_{0}, 0\right) \psi_{0}\right\rangle \neq 0$,

(4) For all $\alpha \in \mathbb{R}, F\left(\lambda, e^{i \alpha} \psi\right)=e^{i \alpha} F(\lambda, \psi)$.

(5) For all $\psi \in X,\langle\psi, F(\lambda, \psi)\rangle \in \mathbb{R}$.

Then $\left(\lambda_{0}, 0\right)$ is a bifurcation point of the equation $F(\lambda, \psi)=0$, in the sense that there is a family of non-trivial solutions, $\left(\lambda_{s}, \psi_{s}\right)$, for $s \in[0, \sqrt{\epsilon})$, unique modulo the global gauge symmetry (apart from the trivial solution $(1,0)$ ) in a neighbourhood of $\left(\lambda_{0}, 0\right)$ in $\mathbb{R} \times X$. Moreover, this family has the form

$$
\left\{\begin{array}{l}
\lambda=\phi_{\lambda}\left(s^{2}\right), \\
\psi=s \psi_{0}+s \phi_{\psi}\left(s^{2}\right) .
\end{array}\right.
$$

Here $\psi_{0} \in \operatorname{null} \partial_{\psi} F\left(\lambda_{0}, 0\right)$, and $\phi_{\lambda}:[0, \epsilon) \rightarrow \mathbb{R}$ and $\phi_{\psi}:[0, \epsilon) \rightarrow X$ are unique real-analytic functions, such that $\phi_{\lambda}(0)=\lambda_{0}, \phi_{\psi}(0)=0$. 


\section{A. Fixing the Gauge}

We provide here an alternate proof of Proposition 4.1, largely based on ideas in [15]. We begin by defining the function $B: \mathbb{R} \rightarrow \mathbb{R}$ to be

$$
B(\zeta)=\frac{1}{r} \int_{0}^{r} \operatorname{curl} A(\xi, \zeta) d \xi
$$

It is clear that $b=\frac{1}{r \tau_{2}} \int_{0}^{r \tau_{2}} B(\zeta) d \zeta$. A calculation shows that $B\left(\zeta+r \tau_{2}\right)=B(\zeta)$.

We now define $P=\left(P_{1}, P_{2}\right): \mathbb{R}^{2} \rightarrow \mathbb{R}^{2}$ to be

$$
P(x)=\left(b x_{2}-\int_{0}^{x_{2}} B(\zeta) d \zeta, \int_{\frac{\tau_{1}}{\tau_{2}} x_{2}}^{x_{1}} \operatorname{curl} A\left(\xi, x_{2}\right) d \xi+\frac{\tau \wedge x}{\tau_{2}} B\left(x_{2}\right)\right) .
$$

A calculation shows that $P$ is doubly-periodic with respect to $\mathcal{L}$.

We now define $\eta^{\prime}: \mathbb{R}^{2} \rightarrow \mathbb{R}$ to be

$$
\eta^{\prime}(x)=\frac{b}{2} x_{1} x_{2}-\int_{0}^{x_{1}} A_{1}(\xi, 0) d \xi-\int_{0}^{x_{2}} A_{2}\left(x_{1}, \zeta\right)-P_{2}\left(x_{1}, \zeta\right) d \zeta .
$$

$\eta^{\prime}$ satisfies $\nabla \eta^{\prime}=-A+A_{0}+P$ and let $\eta^{\prime \prime}$ be a doubly-periodic solution of the equation $\Delta \eta^{\prime \prime}=-\operatorname{div} P$. Also let $C=\left(C_{1}, C_{2}\right)$ be given by

$$
C=-\frac{1}{|\Omega|} \int_{\Omega}\left(P+\nabla \eta^{\prime \prime}\right) d x
$$

where $\Omega$ is any fundamental cell, and set $\eta^{\prime \prime \prime}=C_{1} x_{1}+C_{2} x_{2}$ and define $\eta=\eta^{\prime}+\eta^{\prime \prime}+\eta^{\prime \prime \prime}$.

We claim that $\alpha^{\prime}:=A+\nabla \eta-A_{0}^{b}$ satisfies (i) - (iii) of the proposition. We first note that $\alpha^{\prime}=$ $P+\nabla \eta^{\prime \prime}+C$ and by the above, it is periodic. We also calculate that $\operatorname{div} \alpha^{\prime}=\operatorname{div} P+\Delta \eta^{\prime \prime}=0$. Finally $\int_{\Omega} \alpha^{\prime}=\int_{\Omega}\left(P+\nabla \eta^{\prime \prime}-C\right)=0$.

We note that $A_{0}^{b}(x)+\alpha^{\prime}(x)$ satisfies

$$
A_{0}^{b}(x+t)+\alpha^{\prime}(x+t)=A_{0}^{b}(x)+\alpha^{\prime}(x)+\frac{b}{2}\left(\begin{array}{c}
-t_{2} \\
t_{1}
\end{array}\right) .
$$

We note that $\left(\begin{array}{c}-t_{2} \\ t_{1}\end{array}\right)=\nabla(t \wedge x)$, and therefore, by gauge-periodicity of the pair $\left(e^{\eta} \Psi, A_{0}^{b}+\alpha^{\prime}\right)$, we have that $\Psi^{\prime}:=e^{i \eta} \Psi$ satisfies $\Psi^{\prime}(x+t)=e^{g_{t}(x)} \Psi^{\prime}(x)$, where $g_{t}(x)=\frac{b}{2} t \wedge x+C_{t}$ for some constant $C_{t}$. To establish (i), we need to have it so that $C_{t}=0$ for $t=r, r \tau$. First let $l$ be such that $r \wedge l=-\frac{C_{r}}{b}$ and $r \tau \wedge l=-\frac{C_{r \tau}}{b}$.

This $l$ exists as it is the solution to the matrix equation

$$
\left(\begin{array}{cc}
0 & r \\
-r \tau_{2} & r \tau_{1}
\end{array}\right)\left(\begin{array}{l}
l_{1} \\
l_{2}
\end{array}\right)=\left(\begin{array}{c}
-\frac{C_{r}}{b} \\
-\frac{C_{r \tau}}{b}
\end{array}\right)
$$

and the determinant of the matrix is just $r^{2} \tau_{2}$, which is non-zero because $(r, 0)$ and $r \tau$ form a basis of the lattice. Let $\zeta(x)=\frac{b}{2} x \wedge l$. A straightforward calculation then shows that $\phi(x):=e^{i \zeta(x)} \Psi^{\prime}(x+l)$ satisfies (i) and that $\alpha(x):=\alpha^{\prime}(x+l)+\nabla \zeta(x)+A_{0}^{b} l=\alpha^{\prime}(x+l)$ still satisfies (i) through (iii). This proves the proposition.

Acknowledgements. The first author is supported by the European Research Council under the European Community's Seventh Framework Program (FP7/2007-2013)/ERC grant agreement 202859, and in part by Ontario graduate fellowship and by NSERC under Grant NA 7901. He would also like to express his thanks to Sergey Morozov for his comments and discussions. The second author is suppoted by NSERC Grant NA7601. He is grateful to Yuri Ovchinnikov for many fruitful discussions. A part of this work was done during I.M.S.'s stay at the IAS, Princeton. The authors are grateful to Panayotis Smyrnelis, who red the manuscript and pointed out a number of typographical errors and an erroneous argument. 


\section{References}

[1] A. A. Abrikosov. On the magnetic properties of superconductors of the second group. J. Explt. Theoret. Phys. (USSR) 32 (1957), 1147-1182.

[2] A. Aftalion, X. Blanc, F. Nier. Lowest Landau level functional and Bargmann spaces for Bose-Einstein condensates. J. Funct. Anal. 241 (2006), 661-702.

[3] A. Aftalion, S. Serfaty. Lowest Landau level approach in superconductivity for the Abrikosov lattice close to $H_{c_{2}}$. Selecta Math. (N.S.) 13 (2007), 183-202.

[4] L. V. Alfors. Complex analysis. McGraw-Hill, New York, 1979.

[5] Y. Almog. On the bifurcation and stability of periodic solutions of the Ginzburg-Landau equations in the plane. SIAM J. Appl. Math. 61 (2000), 149-171.

[6] Y. Almog, Abrikosov lattices in finite domains. Commun. Math. Phys. 262 (2006), 677-702.

[7] A. Ambrosetti, G. Prodi. A Primer of Nonlinear Analysis. Cambridge University Press, Cambridge, 1993.

[8] E. Barany, M. Golubitsky, J. Turski. Bifurcations with local gauge symmetries in the Ginzburg-Landau equations. Phys. D 56 (1992), 36-56.

[9] M.S. Berger, Y. Y. Chen. Symmetric vortices for the nonlinear Ginzburg-Landau equations of superconductivity, and the nonlinear desingularization phenomenon. J. Fun. Anal. 82 (1989) 259-295.

[10] S. J. Chapman. Nucleation of superconductivity in decreasing fields. European J. Appl. Math. 5 (1994), $449-468$.

[11] S. J. Chapman, S. D. Howison, J. R. Ockedon. Macroscopic models of superconductivity. SIAM Rev. 34 (1992), 529-560.

[12] Q. Du, M. D. Gunzburger, J. S. Peterson. Analysis and approximation of the Ginzburg-Landau model of superconductivity. SIAM Rev. 34 (1992), 54-81.

[13] M. Dutour. Phase diagram for Abrikosov lattice. J. Math. Phys. 42 (2001), 4915-4926.

[14] M. Dutour. Bifurcation vers l'état d'Abrikosov et diagramme des phases. Thesis Orsay, http://www.arxiv.org/abs/math-ph/9912011.

[15] G. Eilenberger, Zu Abrikosovs Theorie der periodischen Lösungen der GL-Gleichungen für Supraleiter 2. Z. Physik $180(1964), 32-42$.

[16] S. Fournais, B. Helffer. Spectral Methods in Surface Superconductivity. Progress in Nonlinear Differential Equations and their Applications, vol. 77, Birkhäuser, 2010.

[17] S. Gustafson, I.M. Sigal. The stability of magnetic vortices. Comm. Math. Phys. 212 (2000) 257-275.

[18] S. Gustafson, I. M. Sigal. Mathematical Concepts of Quantum Mechanics. Springer, 2006.

[19] S. J. Gustafson, I. M. Sigal, T. Tzaneteas. Statics and dynamics of magnetic vortices and of Nielsen-Olesen (Nambu) strings. J. Math. Phys. 51, 015217 (2010).

[20] A. Jaffe, C. Taubes. Vortices and Monopoles: Structure of Static Gauge Theories. Progress in Physics 2. Birkhäuser, Boston, Basel, Stuttgart, 1980.

[21] W.H. Kleiner, L. M. Roth, S. H. Autler. Bulk solution of Ginzburg-Landau equations for type II superconductors: upper critical field region. Phys. Rev. 133 (1964), A1226-A1227.

[22] G. Lasher. Series solution of the Ginzburg-Landau equations for the Abrikosov mixed state. Phys. Rev. 140 (1965), A523-A528.

[23] S. Nonnenmacher, A. Voros. Chaotic eigenfunctions in phase space. J. Statist. Phys. 92 (1998), 431-518.

[24] F. Odeh, Existence and bifurcation theorems for the Ginzburg-Landau equations. J. Math. Phys. 8 (1967), $2351-2356$.

[25] Yu. N. Ovchinnikov. Structure of the supercponducting state near the critical fiel $H_{c 2}$ for values of the Ginzburg-Landau parameter $\kappa$ close to unity. JETP. 85 (4) (1997), 818-823.

[26] J. Rubinstein. Six Lectures on Superconductivity. Boundaries, interfaces, and transitions (Banff, AB, 1995), 163-184, CRM Proc. Lecture Notes, 13, Amer. Math. Soc., Providence, RI, 1998.

[27] E. Sandier, S. Serfaty. Vortices in the Magnetic Ginzburg-Landau Model. Progress in Nonlinear Differential Equations and their Applications, vol.1 70, Birkhäuser, 2007.

[28] P. Takáč. Bifurcations and vortex formation in the Ginzburg-Landau equations. Z. Angew. Math. Mech. 81 (2001), 523-539.

[29] T. Tzaneteas, I. M. Sigal. Abrikosov lattice solutions of the Ginzburg-Landau equations. Contemporary Mathematics $535,195-213,2011$. 